\title{
Comparison of Pilates Exercises and Proprioceptive Exercises on Joint Position Sense in People with Knee Osteoarthritis
}

\author{
Kaur Rajinder ${ }^{1}$, Kaur Harneet ${ }^{2}$ \\ ${ }^{1}$ Lovely Professional University, Department of Physiotherapy, Jalandhar Phagwara GT Road, Punjab, India \\ ${ }^{2}$ Lovely Professional University, Department of Physiotherapy, Jalandhar Phagwara GT Road, Punjab, India
}

\begin{abstract}
Purpose: Individuals with knee Osteoarthritis suffers from pain, joint stiffness, decreased muscle strength and proprioceptive deficits. Long term physical exercise improves Proprioception and neuromuscular control. Method: 60 subjects with bilateral knee osteoarthritis were randomly divided into 2 groups. Subjects in group A performed pilates exercise and group B performed Proprioceptive exercise for 6 weeks. Knee joint proprioception, Pain and functional disability changes from the baseline were measured. Data was analysed by using paired and unpaired t-test, wilcoxon signed rank test and Mann whitney test. Results: There was significant improvement in knee proprioception, reduction in pain and functional disability in both groups $(p<0.05)$. But the right and left knee proprioception in group $A$ and group B shows significant difference between them with $(p<0.05)$, which indicates that group $A$ had better improvement in knee proprioception The right and left knee pain and functional disability shows no significant difference $(p>0.05)$ between group $A$ and group B. Conclusion: Pilates exercises shows more significant improvement in knee proprioception in people with knee osteoarthritis than the proprioceptive exercises. Also pilates and proprioceptive exercises are equally effective in reduncing pain and improving functional ability in peoples with osteoarthritis
\end{abstract}

Keywords: Osteoarthritis, Pilates exercises, Knee joint position sense, Functional disability.

\section{Introduction}

OA is a frequently slowly progressive joint disease typically seen in middle aged to elderly people.[1] It is estimated that approximately four percent of the world's current population is affected by osteoarthritis.[2] The worldwide prevalence estimated for symptomatic OA is $9.6 \%$ among men and $18 \%$ among women. It is fourth leading cause of year lived with disability (YLD).[3] Knee pain is the most frequently reported peripheral joint complaint in community based studies worldwide and has been found to be present in 5-13\% of adult populations in Asia by the community oriented programme for the control of rheumatic diseases (COPCORD) studies.[2] Common signs and symptoms of knee OA include pain, joint stiffness, decreased muscle strength and proprioceptive deficits. In addition individuals with knee OA often have poor neuromuscular control, slower walking speed, decreased functional ability and increased susceptibility to falling.[4]

Proprioception can be described as a sense of position, which is awareness of position of one's own limb and the orientation of their parts with respect to one another.[5] Adequate proprioception is required for safe and capable movements of the body. Disturbed position sense in lower limbs may lead to perturbation in daily activities such as walking, running and may ultimately lead to injuries. Studies have shown that when the proprioceptive acquity decreases, functional ability can only be attained if there is sufficient muscle strength to compensate for the decrease in accuracy of modulation and activation of muscle.[6] This designates that in the presence of both proprioceptive inaccuracy and muscle weakness, functional ability may be more affected.[7] Proprioceptive and neuromuscular control have shown improvement in healthy individual as a result of long term physical exercise.[8]

So, the rehabilitation for knee OA aims to increase the muscle strength and enhance proprioception function. Several studies have demonstrated the efficacy of proprioception exercises in improving joint position sense in patients with knee $\mathrm{OA}$ by enhancing the sensorimotor mapping of knee movement. A study by DA HON LIN et al has demonstrated that proprioceptive exercises causes greater improvement in knee reposition sense and walking speed on the spongy surface. However there are few reports investigating the benefits of pilates training in patients with knee OA. Pilates, originally developed by Joseph Pilates, is described as " A unique method of physical fitness that uses the combination of muscle strengthening, lengthening and breathing to develop trunk muscles and restore muscle balance". A study by Nursen has shown that pilates exercise training leads to improvement in joint position sense in healthy individuals, but less attempts have been made till date to investigate the efficacy of pilates in improving JPS in patients with knee OA. This arises a need to find out which one (pilates training or proprioception training) is a better treatment protocol in improving joint position sense in people with knee osteoarthritis. So the primary objective of this study was to compare the effectiveness of Pilate's exercises and Proprioceptive exercises on knee joint position sense in people with knee osteoarthritis. Previous studies have suggested that muscle strength and proprioception need to be improved first in order to improve the functional performance. So the secondary objective of this study was to compare the effects of pilates exercise and proprioceptive exercises on pain and functional disability. 


\section{International Journal of Science and Research (IJSR) \\ ISSN (Online): 2319-7064}

Index Copernicus Value (2013): 6.14 | Impact Factor (2014): 5.611

\section{Methodology}

This comparative, randomised experimental study was conducted in accordance with the principles of good clinical practice. The protocol was approved by the Ethics Committee of the Lovely Professional University and the written consent was obtained from all patients. The study consisted of 60 subjects (male, female both) 50-60 years of age affected with bilateral knee OA (grade 1 and 2 radiologically according to Kellgrn Lawrence classification), having pain in both knees from past 3 months and those who can walk on toes and heels. The diagnosis of OA was made on the basis of clinical history, radiographic imaging and physical assessment. The patients suffering from any traumatic injury to hip, knee, ankle and back, vascular injury, neurological injury, psychiatric illness, deformity in lower limbs, leg length discrepancy and inflammatory arthritis of knee joint were excluded from the study. Eligible patients were randomly assigned in a 1:1 ratio into two groups (group A and group B containing 30 subjects each) using a lottery method.

\section{Procedure}

Before the commencement of the study, proprioception was measured in bed sitting position with legs out of the plinth and thigh fully supported using a universal goniometer. With the subject eyes closed, the examiner passively moved the limb to reach the knee joint to 30 degree of flexion which was the test angle. To gain accuracy in measuring the angle, the Goniometer was tied to the lower limb in such a manner that the fulcrum was coinciding with the lateral knee joint line, while one arm of the goniometer was aligned parallel to the line joining greater trochanter and fulcrum and the other arm to the leg i.e. along the line joining fulcrum and lateral malleolus. Subject was blind folded to avoid any visual cues. Then examiner passively flex the knee joint from the extended position to the target angle of 30 degree at very slow speed (about 10 degree/second). Subject attempted to identify test position whilst holding it actively for 4 seconds and then passively returned to the starting position. Five practice sessions were given and then patient was asked to reproduce the target position actively using the same limb. All subjects were given initial explanation and practice before formal examination. Responses position was measured as the angle at which subject stopped. Three consecutive response angles were noted. Functional disability was measured by WOMAC score which was filled by patient. Patients were assisted for understanding the language of WOMAC scale. The pain was assessed by using NPRS.

\section{Protocol}

Group A performed proprioceptive exercises and Group B performed Pilates exercise for three days a week during 8 weeks (Table 1). Pilates exercise program was developed by the researchers of the study, based on Levine et al.'s pilates training. Each session lasted one hour. The class consisted of 20 minutes warm up and cool down (10 minutes before and 10 minutes after exercise) and 40 minutes (increased from gradually from 20 minutes) of pilates exercises.
Table 1: Exercise protocol

\begin{tabular}{|c|c|c|}
\hline & $\begin{array}{c}\text { Group A } \\
\text { (Pilates Exercises) }\end{array}$ & $\begin{array}{c}\text { Group } B \\
\text { (Proprioceptive Exercises) }\end{array}$ \\
\hline Week 1 & $\begin{array}{c}\text { Hundreds } \\
\text { (5 repetitions) }\end{array}$ & $\begin{array}{c}\text {-One -leg balances } \\
\text {-Forward -backward leg swings } \\
\text { with knees flexed } \\
\text {-Forward-backward leg swings } \\
\text { with knee extended } \\
\text {-Toe walking } \\
\text {-Heel walking } \\
\text {-Cross body leg swings }\end{array}$ \\
\hline Week 2 & $\begin{array}{c}\text { week } 1+\text { one leg } \\
\text { stretch, double leg } \\
\text { stretch (6 repetitions) }\end{array}$ & Same as week 1 \\
\hline Week 3 & $\begin{array}{l}\text { week } 2+\text { clams } \\
\text { (7 repetitions) }\end{array}$ & $\begin{array}{c}\text {-Advanced one leg balances } \\
\text {-Maximum forward-backward leg } \\
\text { swings with knee extended } \\
\text {-Toe walking } \\
\text {-Heel walking } \\
\text {-One -leg squats } \\
\text {-Runners poses }\end{array}$ \\
\hline Week 4 & $\begin{array}{c}\text { week } 3+\text { one leg kick } \\
\text { (7 repetitions) }\end{array}$ & Same as week 3 \\
\hline Week 5 & $\begin{array}{l}\text { week } 4+\text { side kick } \\
\text { (8 repetitions) }\end{array}$ & \begin{tabular}{|c|}
-Blind advanced one leg balances \\
-Bicycle leg swings \\
-Partial squats \\
-Toe walking \\
-Heel walking \\
\end{tabular} \\
\hline Week 6 & $\begin{array}{c}\text { week } 5+\text { one leg } \\
\text { circle (10 repetitions) }\end{array}$ & Same as week 5 \\
\hline
\end{tabular}

\section{Statistical Analysis}

Position sense accuracy was measured as the arithmatic difference between test and response angle. Baseline values of pain and proprioception were compared between the groups by using unpaired t-test. For comparing the pre and post values for pain and proprioception within the groups paired t-test was used while for comparing the values between the two groups unpaired t-test was used. Baseline values for WOMAC were compared between the groups by using Mann whitney test. The pre and post values for WOMAC were compared within the groups by using Wilcoxon Signed Rank test whereas the WOMAC scores were compared between the groups by using Mann Whitney test.

\section{Result}

There was decrease in pain score in both groups i.e. in group A (from 5.67 to 4.63 for right knee and from 5.30 to 4.73 for left knee) and group B (from 5.53 to 4.67 for right knee and from 5.17 to 4.23 for left knee), which shows significant difference in pre and post values within the groups $(\mathrm{p}<0.05)$ (Table 3) (Figure1 A and B). There was significant improvement in proprioception (response angle) in both the groups i.e in group A (from 26.92 to 30.05 for right knee and from 29.92 to 30.15 for left knee) and group B (from 26.67 to 28.03 for right knee and from 26.86 to 27.98 for left knee) with $\mathrm{p}<0.05$ (Table 3) (Figure I D and E). The WOMAC scores were significantly improved in both the groups (group A from 69.72 to 33.11 and group B from 69.43 to 35.47 ) (Table 3) (Figure I C). The comparison between right knee pain and left knee pain in group A and group B shows no 


\section{International Journal of Science and Research (IJSR) \\ ISSN (Online): 2319-7064 \\ Index Copernicus Value (2013): 6.14 | Impact Factor (2014): 5.611}

significant difference $(\mathrm{p}>0.05)$ (Table 4). The comparison between the right and left knee proprioception in group A and group B shows significant difference between them $(\mathrm{p}<$ 0.05), which indicates that group A had better improvement in knee proprioception of both knees than group B (Table 4). The comparison between WOMAC scores between group A and group $B$ shows no significant difference between them (p>0.05) (Table 4).

\section{Discussion}

A total of 60 subjects suffering from bilateral knee OA (grade 1 and 2 radiologically according to Kellgrn Lawrence classification) participated and completed this comparative study. In this study it was found that pilates exercises and proprioceptive exercises done for 1 hour for 6 weeks is helpful in improving proprioception, decreasing pain and improving functional ability in patients suffering from knee OA. Though greater improvement in proprioception was seen in subjects who performed pilates exercises. Our findings are consistent with the results of previous studies. But our study is the first to demonstrate that pilates exercises are more beneficial in improving the joint proprioception as compared to the proprioceptive exercises.

There are various mechanisms that support the present study. Regular physical activity and exercise generates an impressive collection of favourable effects in many physiologic systems. Several mechanisms could be pointed to explain the positive impact of regular physical activity and exercise on joint proprioception. It has been suggested that central and peripheral components of proprioception implicated in the age related decline on proprioception function are potentially related to its improvement after regular physical exercise.[9]

Physical exercise does not change the number of mechanoreceptors but induces morphological adaptations in the muscle spindle at the peripheral level. There are muscle spindle adaptations on a microlevel, the intrafusal muscle fibres could show some metabolic changes and on a macrolevel, the latency of stretch reflex responses decreases and the amplitude increases. At central level, regular physical activity and exercise is able to change proprioception through the modulation of muscle spindle gain and the induction of plastic modifications in central nervous system. During the physical activities an increase in the muscle spindle output through the gamma route is observed, which facilitates the cortical projection of proprioception. Thus, by increasing the output of the muscle spindle over time, it is possible to induce plastic changes in the central nervous system, such as increased strength of synaptic connections and/or structural changes in the organization and number of connections among neurons. These repetitive afferent inputs from mechanoreceptors induce plastic changes in the cortex would modify the cortical maps of the body overtime, increasing the cortical representation of the joints and leading to enhanced joint proprioception.[9]
Table 2: Baseline characteristics of the patients

\begin{tabular}{|c|c|c|}
\hline Parameters & Group A & Group B \\
\hline Age (Years) & $55.97 \pm 3.296$ & $55.50 \pm 3.256$ \\
\hline Height (Inches) & $66.00 \pm 3.591$ & $65.80 \pm 2.952$ \\
\hline Weight (Kgs) & $76.83 \pm 5.896$ & $74.47 \pm 6.388$ \\
\hline BMI (Kg/m ${ }^{2}$ ) & $27.31 \pm 1.804$ & $26.57 \pm 1.607$ \\
\hline Pain (NPRS) & & \\
Right Knee & $5.67 \pm 1.295$ & $5.53 \pm 1.456$ \\
Left Knee & $5.30 \pm 0.99$ & $5.17 \pm 1.29$ \\
\hline Response Angle (degrees) & & \\
Right Knee & $26.92 \pm 0.846$ & $26.67 \pm 1.229$ \\
Left Knee & $29.92 \pm 1.016$ & $26.86 \pm 0.980$ \\
\hline Functional Disability & & \\
(WOMAC) & $69.72 \pm 7.330$ & $69.43 \pm 8.111$ \\
\hline
\end{tabular}

Table 3: Comparison of the variables within the groups

\begin{tabular}{|c|c|c|c|}
\hline Variables & Baseline & After 6 weeks & $P$ value \\
\hline Group A & & \\
\hline Response Angle (degrees) & & & \\
Right knee & $26.92 \pm 0.846$ & $30.05 \pm 0.655$ & $0.000^{*}$ \\
Left knee & $29.92 \pm 1.016$ & $30.15 \pm 0.559$ & $0.000^{*}$ \\
\hline Pain & $5.67 \pm 1.295$ & $4.63 \pm 1.066$ & $0.000^{*}$ \\
Right knee & $5.30 \pm 0.99$ & $4.73 \pm 1.17$ & $0.000^{*}$ \\
Left knee & $69.72 \pm 7.330$ & $33.11 \pm 5.008$ & $<0.001^{*}$ \\
\hline WOMAC & Group B \\
\hline \multicolumn{4}{|c|}{} \\
\hline Response Angle (degrees) & $26.67 \pm 1.229$ & $28.03 \pm 0.563$ & $0.000^{*}$ \\
Right knee & $26.86 \pm 0.980$ & $27.98 \pm 0.623$ & $0.000^{*}$ \\
Left knee & $5.53 \pm 1.456$ & $4.67 \pm 1.061$ & $0.000^{*}$ \\
\hline Pain & $5.17 \pm 1.29$ & $4.23 \pm 1.10$ & $0.000^{*}$ \\
\hline Right knee & $69.43 \pm 8.111$ & $35.47 \pm 5.424$ & $<0.001^{*}$ \\
\hline Left knee & \multicolumn{3}{|}{}
\end{tabular}

(Note * indicates $\mathrm{p}<0.05$ )

Table 4: Comparison of the parameters between the groups

\begin{tabular}{|c|c|c|c|c|c|}
\hline Parameters & \multicolumn{2}{|c|}{ Group A } & \multicolumn{2}{c|}{ Group B } & $P$ \\
\hline & Pre & Post & Pre & Post & \\
\hline Response Angle (degrees) & & & & & \\
Right knee & 26.92 & 30.05 & 26.67 & 28.03 & $0.000^{*}$ \\
Left knee & 29.92 & 30.15 & 26.86 & 27.98 & $0.000^{*}$ \\
\hline Pain & & & & & \\
Right knee & 5.67 & 4.63 & 5.53 & 4.67 & 0.9038 \\
Left knee & 5.30 & 4.73 & 5.17 & 4.23 & 0.944 \\
\hline WOMAC & 69.72 & 33.11 & 69.43 & 35.47 & 0.062 \\
\hline
\end{tabular}

(Note * indicates $p<0.05)$

\section{Figures}

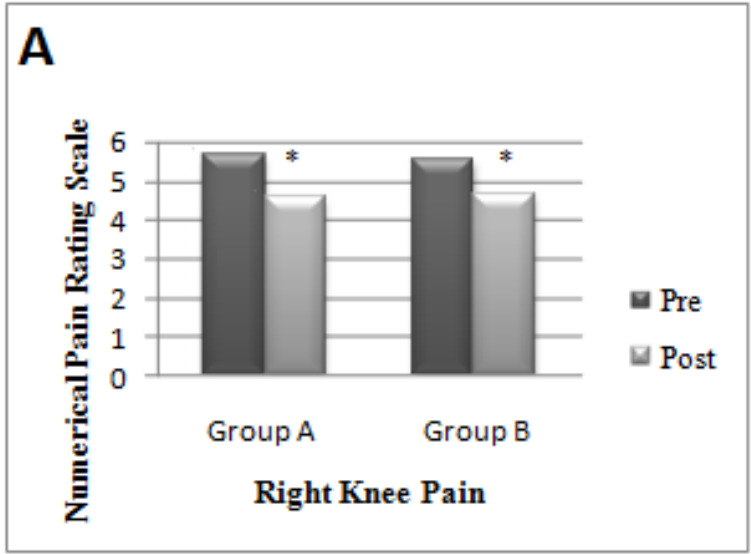

Figure 1: 

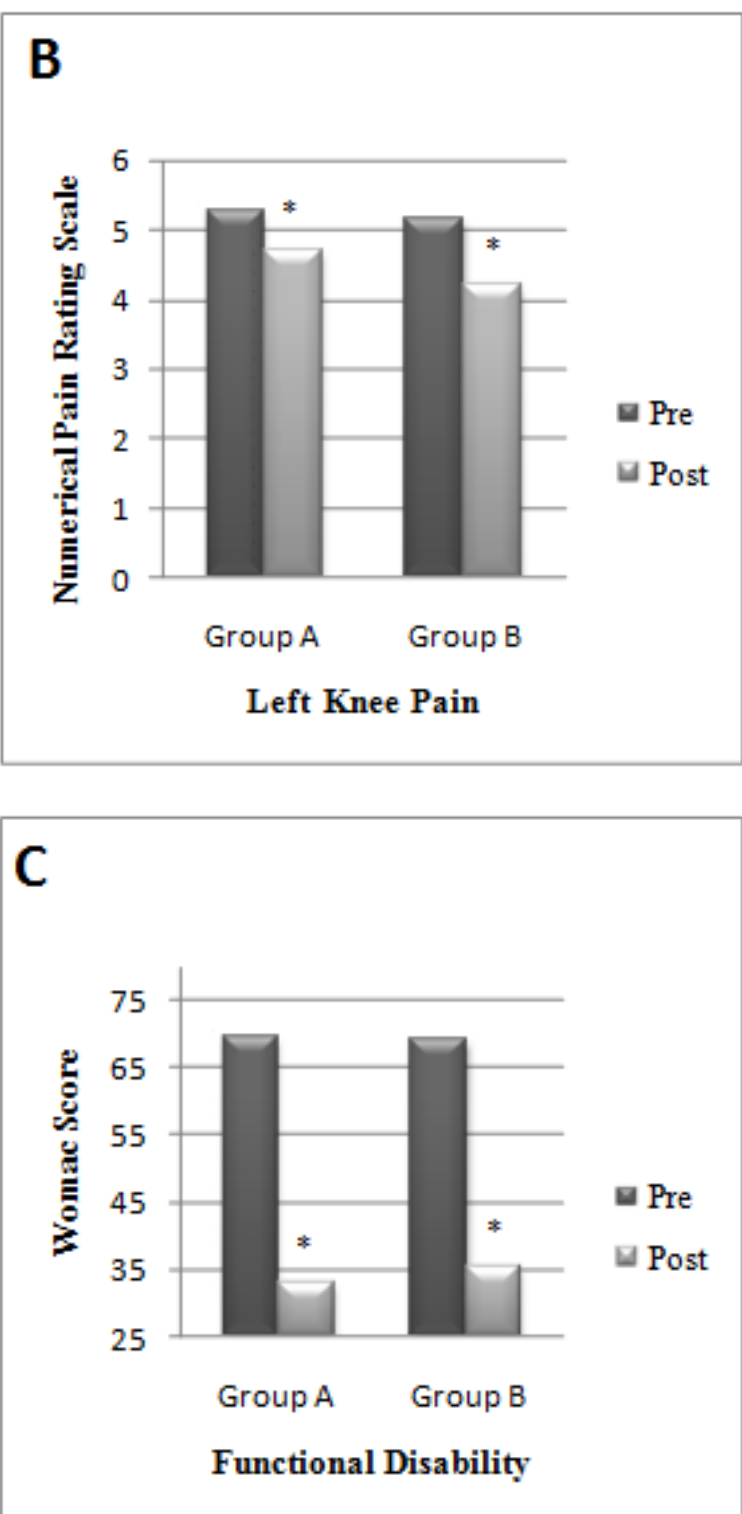

\section{D}

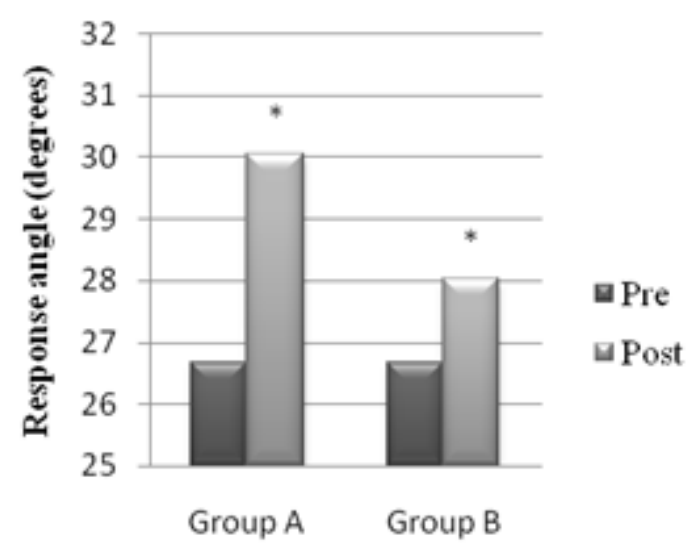

Right Knee Proprioception

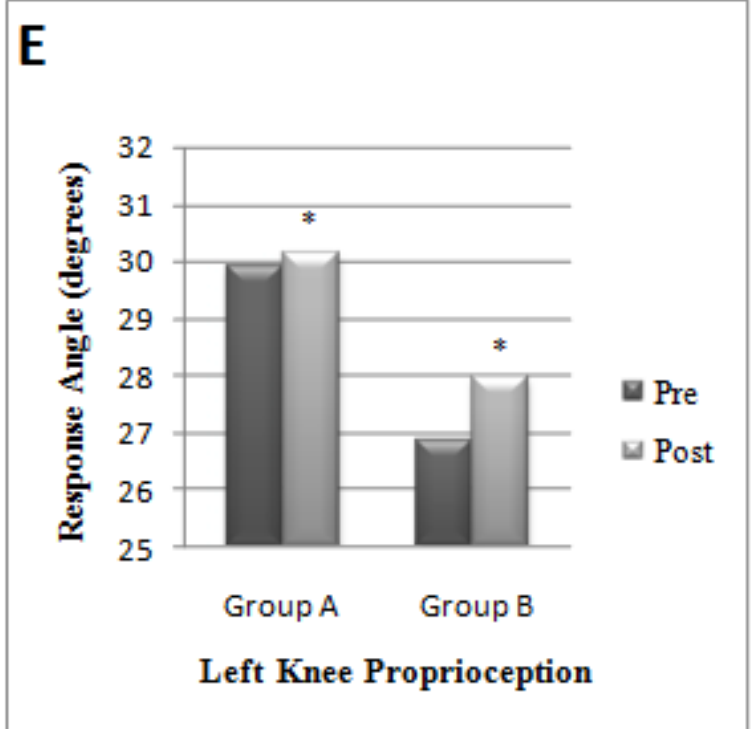

Figure 1: Effect of pilates and proprioceptive exercises on A. Right Knee Pain, B. Left Knee Pain, C. Functional Disability, D. Right Knee Proprioception (at target position $30^{\circ}$ ), E. Left Knee Proprioception (at target position $30^{\circ}$ ).

(Note: * indicates $\mathrm{p}<0.05$ )

In the present study, Pilates's exercises were given in the group A for 6 weeks, 3 times in a week. The results showed significant improvement in knee joint proprioception of both knees in patients with knee osteoarthritis. Pilate's method is a new approach for clinical setting. Pilate's method is defined as comprehensive body-mind conditioning, with main goals which are efficient movement, core stability and enhanced performance. The pilate's exercise improves physical and mental conditioning through increasing strength, flexibility, balance and postural awareness by stretching and strengthening exercises.[6] Secondly, the pilates method focuses on building motions and activities that help to strengthen minor muscles, which in turn, helps to strengthen major muscles. Thirdly, pilates exercise may affect proprioception via mental effort, focuses on activating specific muscles at correct speed, quality, precision and control of movement with specific joint awareness.[6]

A previous study shows that proprioception exercises are superior to conventional treatment in terms of increasing range of motion, decrease in pain NPRS, and decreasing disability WOMAC score. Proprioceptive exercises improves adequate motor skills for delaying with potential destabilising force on the knee that increased activities of daily living.[10]

Several other studies have investigated the effects of different type of exercises on the restoration of proprioception in knee injuries. A study by Subasi et al reported that warm up exercises improve knee position sense. They pointed out that warm up exercises may improve mechanoreceptors sensitivity by improving proper viscoelastic properties of the muscle tissue, enhanced oxygenation, and increased body temperature caused by vasodilatation. According to Boute et al, these alterations may improve the functioning of these receptors and kinaesthetic sensibility. Increased temperature decreases the threshold of the mechanoreceptors and improves tactile sensibility by exercises. The muscular 


\section{International Journal of Science and Research (IJSR) \\ ISSN (Online): 2319-7064}

Index Copernicus Value (2013): 6.14 | Impact Factor (2014): 5.611

response probably differs after the exercise, this could be affected by a modification of either corollary discharges likely involved in position sense, fusimotor commands and spindle activity. Therefore, muscular exercise improves proprioception by enhancing motor performances due to not only the improved mechanical properties of the muscles but also to better kinaesthetic sensibility.[6]

In this study there was significant reduction WOMAC score in both the groups. It has suggested that pain is related to functional performance but is not proportional to it.[11] It has been reported that an increase in muscle strength of elderly is a major factor for improving functional performance as the weakening of the quadriceps femoris muscle in degenerative osteoarthritis patients decreases functional performance and thus determines the severity of disability.[12] According to previous research, the weakening of femoris muscle correlates with a decrease in joint position sense and a degradation of functional performance. Furthermore, weakening of muscle strength and degradation of proprioception both affect the functional performance. These studies suggest that muscle strength and proprioception need to be improved first, in order to improve functional performance.[13]

So in this study knee proprioception was improved, which improved functional performance in the patients with knee osteoarthritis.

In conclusion this study shows that both pilates and proprioceptive exercises helps in improving knee proprioception, pain and reducing functional disability. But, there was more significant improvement in joint position sense of group A (Pilates exercises) than group B (Proprioceptive exercises), which shows that Pilates exercises are better exercises for improving knee joint proprioception in patients with knee osteoarthritis. The limitations of this study included small sample size, short duration of the study and no follow up was taken.

\section{Future Scope}

Further studies can be done including large sample size and the follow up can be taken. This study can be done on geriatric population. All the stages of knee osteoarthritis can be taken and the effect of Pilates and Proprioceptive exercises can be seen on those patients. Acute stages of knee osteoarthritis can be taken.

\section{References}

[1] Thitinan Srikulmontree. American College of Rheumatology. Available http://www.rheumatology.org/I-Am-A/PatientCaregiver/Diseases-Conditions/Osteoarthritis. [Accessed on Jan, 29, 2014]

[2] Vijay Batra, Vijai Prakash Sharma, Meenakshi Batra et al. CIFKAS A Measurer of Functional Disability Status in Knee Osteoarthritis. "Internet Journal of Medical Update", 7(1), pp 47-51, 2012.
[3] Pushpa S Patil, Umesh R Dixit, Chidendra M Shettar. "IOSR Journal of Dental and Medical Sciences", 2(5), pp. 8-10, 2012.

[4] Da Hon Lin, Chein-Ho-Janice, Yong-Fwu Lin et al. Efficacy of 2 Non-Weight Bearing Interventions, Proprioception Training Versus Strength Training, for Patients With Knee Osteoarthritis: A Randomised Clinical Trial. "Journal of Orthopaedic \& Sports Physical Therapy", 39(6), pp. 450-457, 2009.

[5] Schmidt RF, Altner H. Fundamentals of sensory physiology, Robert 3rd edition, Springer-Verlag, 47, 1986.

[6] The Effects of Pilates Exercise Training on Knee Proprioception- A Randomised Controlled Trial. "ĐLT 23, SAYI 2, (MAYIS)", pp. 71-79, 2009.

[7] Takashi Nagai, Timothy C Sell, Scott M Lephart. Effect of Age and Osteoarthritis on Knee Proprioception. "US Musculoskeletal Review", pp. 69-70, 2007.

[8] Stratford PW, Kennedy DM. Performance measures were necessary to obtain a complete picture of osteoarthritic patients. "Journal of Clinical Epidemiology", 59(2), pp. 160-167, 2006.

[9] Fernando Riberio and Jose Oliveria. Effect of physical exercise and age on knee joint position sense. "Archives of Gerontology and Geriatrics", 51, pp 64-67, 2010.

[10] Srinivas Mondam, Srikanth Babu V, Ravindra Kumar B. A Comparative Study of Proprioceptive Exercises versus Conventional Training Programon Osteoarthritis of Knee. "Research Journal of Recent Sciences". 1(12), pp. 31-35, 2012.

[11] Hurley MV, Rees J, Newham DJ. Quadriceps function, proprioceptive acuity and functional performance in healthy young, middle-aged and elderly subjects. "Age and ageing" 27(1), pp. 55-62, 1998.

[12] Hurley MV, Scott DL. Improvements in quadriceps sensorimotor function and disability of patients with knee osteoarthritis following a clinically practicable exercise regime. "British Journal of Rheumatology", 37(11), pp. 1181-1187, 1998.

[13] Taesung Ko, Sukmin Lee, Dongiin Lee. Manual Therapy and Exercise for OA Knee: Effects on Muscle Strength, Proprioception, and Functional Performance. "Journal of Physical Therapy Science", 21(4), pp. 293-299, 2009.

\section{Authors Profile}

Rajinder Kaur received BPT and MPT (Orthopaedics) degree from Lovely Professional University, Punjab in 2012 and 2014.

Harneet Kaur received BPT and MPT (Cardiopulmonary) degree from Lovely Professional University, Punjab in 2012 and 2014. She is currently working as Assistant Professor in Department of Physiotherapy with Khalsa College, Amritsar, Punjab. 